ARTICLE

\title{
CD24 tracks divergent pluripotent states in mouse and human cells
}

Nika Shakiba1, Carl A. White ${ }^{1,2}$, Yonatan Y. Lipsitz ${ }^{1}$, Ayako Yachie-Kinoshita1,2, Peter D. Tonge ${ }^{3}$, Samer M.I. Hussein ${ }^{3}$, Mira C. Puri ${ }^{3,4}$, Judith Elbaz ${ }^{3}$, James Morrissey-Scoot ${ }^{1}$, Mira Li ${ }^{3}$, Javier Munoz ${ }^{5,6}$, Marco Benevento ${ }^{5,6}$, lan M. Rogers $3,7,8$, Jacob H. Hanna9 ${ }^{9}$ Albert J.R. Heck ${ }^{5,6}$, Bernd Wollscheid ${ }^{10,11,12}$, Andras Nagy ${ }^{3,8,13} \&$ Peter W. Zandstra ${ }^{1,2}$

Reprogramming is a dynamic process that can result in multiple pluripotent cell types emerging from divergent paths. Cell surface protein expression is a particularly desirable tool to categorize reprogramming and pluripotency as it enables robust quantification and enrichment of live cells. Here we use cell surface proteomics to interrogate mouse cell reprogramming dynamics and discover CD24 as a marker that tracks the emergence of reprogramming-responsive cells, while enabling the analysis and enrichment of transgenedependent (F-class) and -independent (traditional) induced pluripotent stem cells (iPSCs) at later stages. Furthermore, CD24 can be used to delineate epiblast stem cells (EpiSCs) from embryonic stem cells (ESCs) in mouse pluripotent culture. Importantly, regulated CD24 expression is conserved in human pluripotent stem cells (PSCs), tracking the conversion of human ESCs to more naive-like PSC states. Thus, CD24 is a conserved marker for tracking divergent states in both reprogramming and standard pluripotent culture.

\footnotetext{
${ }^{1}$ Institute of Biomaterials and Biomedical Engineering (IBBME), University of Toronto, Toronto, Ontario, Canada M5S 3E1. ${ }^{2}$ The Donnelly Centre for Cellular and Biomolecular Research (CCBR), University of Toronto, Toronto, Ontario, Canada M5S 3E1. ${ }^{3}$ Lunenfeld-Tanenbaum Research Institute, Mount Sinai Hospital, Toronto, Ontario, Canada M5G 1X5. ${ }^{4}$ Department of Medical Biophysics, University of Toronto, Toronto, Ontario, Canada M5T 3H7. ${ }^{5}$ Biomolecular Mass Spectrometry and Proteomics, Bijvoet Center for Biomolecular Research and Utrecht University for Pharmaceutical Sciences, Utrecht University, Padualaan 8, 3584 CH Utrecht, The Netherlands. ${ }^{6}$ Netherlands Proteomics Centre, Padualaan 8, $3584 \mathrm{CH}$ Utrecht, The Netherlands. ${ }^{7}$ Department of Physiology, University of Toronto, Toronto, Ontario, Canada M5S 1A8. ${ }^{8}$ Department of Obstetrics and Gynecology, University of Toronto, Toronto, Ontario, Canada M5G 1E2. ${ }^{9}$ The Department of Molecular Genetics, Weizmann Institute of Science, Rehovot, Israel 76100. ${ }^{10}$ Department of Biology, Institute of Molecular Systems Biology, Swiss Federal Institute of Technology (ETH) Zürich, Wolfgang-Pauli Strasse 16, 8093 Zürich, Switzerland. ${ }^{11}$ NCCR Neuro Center for Proteomics, University and Swiss Federal Institute of Technology (ETH) Zürich, Wolfgang-Pauli Strasse 16, 8093 Zurich, Switzerland. ${ }^{12}$ Department of Health Sciences and Technology, Swiss Federal Institute of Technology (ETH) Zürich, Universitätstrasse 2, 8092 Zurich, Switzerland. ${ }^{13}$ Institute of Medical Science, University of Toronto, Toronto, Ontario, Canada M5T 3H7. Correspondence and requests for materials should be addressed to P.W.Z. (email: peter.zandstra@utoronto.ca).
} 
E xogenous overexpression of four key transcription factorsOct4, Klf4, c-Myc and Sox2 (OKMS) -allows somatic cells to be induced to a pluripotent state ${ }^{1,2}$. The induced pluripotent stem cells (iPSCs) that emerge as a result of reprogramming are able to contribute to all three germ layers and give rise to an adult organism ${ }^{1}$. Analysis of the reprogramming time course has revealed checkpoints through which cells traverse at the genomic ${ }^{3,4}$, proteomic ${ }^{5,6}$ and epigenetic ${ }^{4}$ levels to achieve a final iPSC state. In the mouse system, SSEA1 is a widely used marker to track the initiation of reprogramming, Nanog and Oct4 for maturation, and Pecam 1 to signify stabilization in an iPSC state ${ }^{3,4}$. Important hallmarks of successful reprogramming include the ability to silence transgenes and the ability to give rise to all germ layers on differentiation ${ }^{1,7,8}$.

While surrogate markers have been used to track the emergence of embryonic stem cell (ESC)-like iPSCs during reprogramming, not all cells traverse common checkpoints to attain a final transgene-independent pluripotent cell state ${ }^{4,8}$. In fact, it has been shown that OKMS factor expression levels play a role in directing cell fate changes during reprogramming. Recently, Tonge et al. ${ }^{9}$ showed that high levels of OKMS expression direct cells along a divergent reprogramming route to produce 'F-class' iPSCs, a novel class of iPSC that is transgenedependent while exhibiting pluripotent properties. This novel state provides insights into the potential spectrum of states emerging as a result of OKMS overexpression while also possessing advantageous properties over traditional, ESC-like cells. F-class iPSCs not only possess a growth advantage over ESC-like iPSCs, but their self-renewal is leukemia inhibitory factor (LIF)-independent, allowing for cost-efficient cell production ${ }^{9}$. Furthermore, F-class cells can be transitioned to an ESC-like iPSC state with the addition of histone deacetylase inhibitors 9 .

With growing knowledge on the role of OKMS expression levels in directing cell fate changes there is a need for easy and reliable benchmarking tools to track cell state during reprogramming. Cell surface proteins are particularly desirable as a tool because of their ability to be quantified with relative ease and without the need to sacrifice cell viability.

As part of the international Project Grandiose initiative ${ }^{10}$, we set out to identify surface glycoproteins emerging during high OKMS factor expression that could capture divergent cell state changes between transgene-dependent (F-class) and ESC-like iPSCs 9 . Our analysis and subsequent validation reveal CD24 as a novel candidate marker that is able to demarcate transgenedependent (F-class) and transgene-independent (ESC-like) mouse iPSCs while identifying 'primed' and 'naive' pluripotent states in transgene-independent populations. Importantly, CD24 exhibits conservation in the human system, tracking the conversion of human ESCs to more naive-like alternative pluripotent states ${ }^{11}$, thus allowing for enrichment of these cells.

\section{Results}

Cell surface proteome dynamics during reprogramming. In order to investigate changes in cell surface glycoproteins resulting in high OKMS factor expression, we employed a secondary $\left(2^{\circ}\right)$, doxycycline (DOX)-inducible mouse embryonic fibroblast (MEF) $1 \mathrm{~B}$ reprogramming system ${ }^{7,10}$. As previously described ${ }^{10}$, cell samples were collected at multiday intervals over the complete reprogramming time course of 30 days (cultured at $1,500 \mathrm{ng} \mathrm{ml}^{-1}$ of DOX and termed the 'DOX-high $(\mathrm{H})$ ' time course), and over one derivate time course (cultured at $5 \mathrm{ng} \mathrm{ml}^{-1}$, termed 'DOX-low (L)'), which was split from the DOX-high time course on day 8 (ref. 10). Samples were then prepared for protein analysis using mass spectrometry through enrichment for cell surface glycopeptides ${ }^{12}$, and labelling of 'full' protein content for quantification ${ }^{13}$, reported by Benevento et al. ${ }^{14}$. A summary of the mass spectrometry pipeline is shown in Supplementary Fig. 1a.

We identified a total of $896 \mathrm{~N}$-glycosylated peptides, representing 432 protein groups (or 'proteoforms' ${ }^{\text {'5 }}$ ) at an estimated confidence level of $98 \%$ (Supplementary Data 1 provides a deconvolution of isoforms). To the best of our knowledge, 34 of these proteoforms have not previously been definitively detected at the cell surface and 86 have not been reported in the context of ESC/iPSC. A list of amino-acid sequences for the 896 detected glycopeptides is provided with sequons highlighted as a resource addressing sites of N-glycosylation (Supplementary Data 2). Panther gene list analysis ${ }^{16,17}$ of the genes corresponding to the full set of detected surface proteins reported their division into eight functional categories (Supplementary Fig. 1b). Receptor, catalytic, binding and transporter activities proved to be dominant. The s.d. of the percentage proportion across the intervals for a given category averaged to $1.5 \%$ for all categories (Supplementary Data 3).

Raw, normalized and averaged spectral counts across three technical replicates per point of the DOX-high time course are presented in Supplementary Data 4. Results are also available on www.stemformatics.org as track files, which enables automated upload to the UCSC genome browser ${ }^{18}$. In order to improve the rigour of the quantitative analysis, we used only that subset of the detected cell surface proteome which overlapped those proteins detected in the 'full' proteome ${ }^{14}$, namely 185 proteoforms, except where otherwise noted. Together, these are listed for further examination as potential surface markers (Supplementary Data 5).

The sharpest degree of protein change at the cell surface occurred between days 0 and 2 (Supplementary Fig. 1c). Downregulation was observed across all of the functional categories (Supplementary Data 6). The distribution of the downregulated proteins among functional categories was congruent to the proportions of the categories for the full list of detected cell surface proteins. Complementary upregulation occurred between day $18(\mathrm{H})$ and the $2^{\circ}$ iPSC state (Supplementary Fig. 1d), and to a lesser extent, between day 21 (DOX-low) and the $2^{\circ}$ iPSC state. Overall, $78 \%$ of the post day 18 upregulated proteins (including most collagens and integrins) were previously downregulated twofold or more (between days 0 and 2). This indicates the impact of transgene activation in directing changes at the cell surface. Interestingly, as compared with the global proteome monitored throughout reprogramming ${ }^{14}$, cell surface glycoproteins are proportionally over-represented among proteins exhibiting twofold or greater change between each time point, and in proteins associated with cell signalling and cell surface receptor expression (Supplementary Data 6). Together, these results underline the importance of utilizing these proteins to track cell state changes.

Consistent with the other 'omic' analyses conducted as part of the Project Grandiose reprogramming analysis ${ }^{10}$, principal component analysis of surface proteins depicted the DOX-high time course diverging from an ESC-like state to a separate state, whereas the DOX-low time course converges on the ESC-like state (Fig. 1a). The divergence suggests the possibility of capturing an alternative reprogramming state, F-class ${ }^{9}$, by means of surface proteomics. We then conducted subsequent analysis to select surface proteins for additional scrutiny and validation (Supplementary Fig. 2). First, we conducted K-means clustering analysis to select genes that are differentially expressed in the F-class state, identifying two clusters of genes that are upregulated following DOX induction and maximally expressed at the F-class state (Supplementary Fig. 2). Of these genes, we selected those with the highest contribution to the principal components separating the F-class and ESC states as well as F-class and 
a

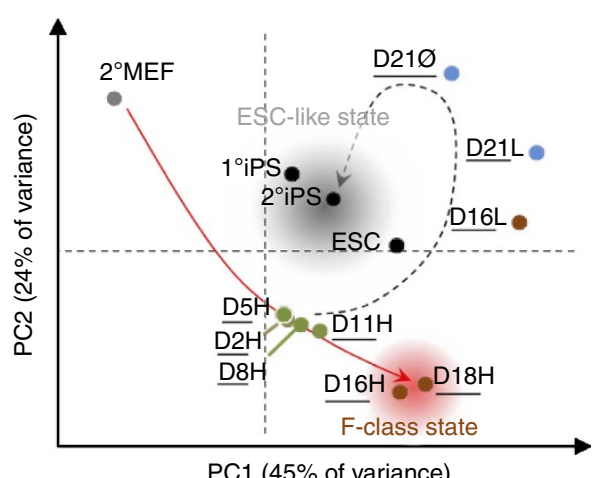

$\mathrm{PC} 1$ (45\% of variance) b

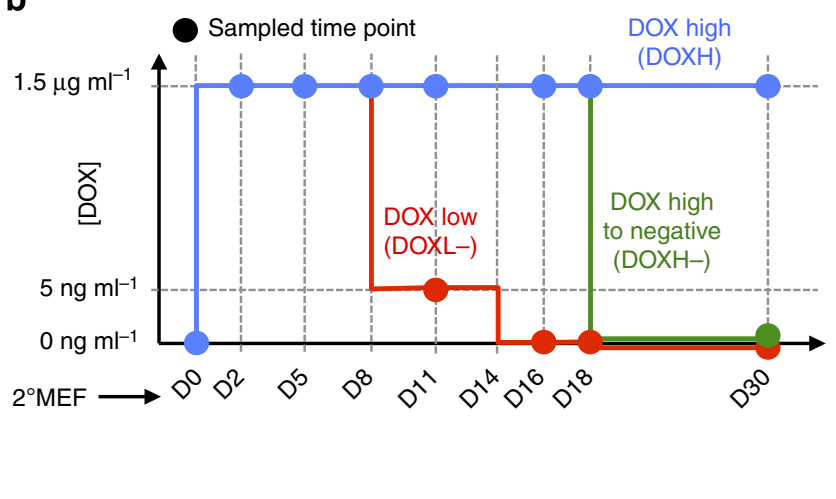

C
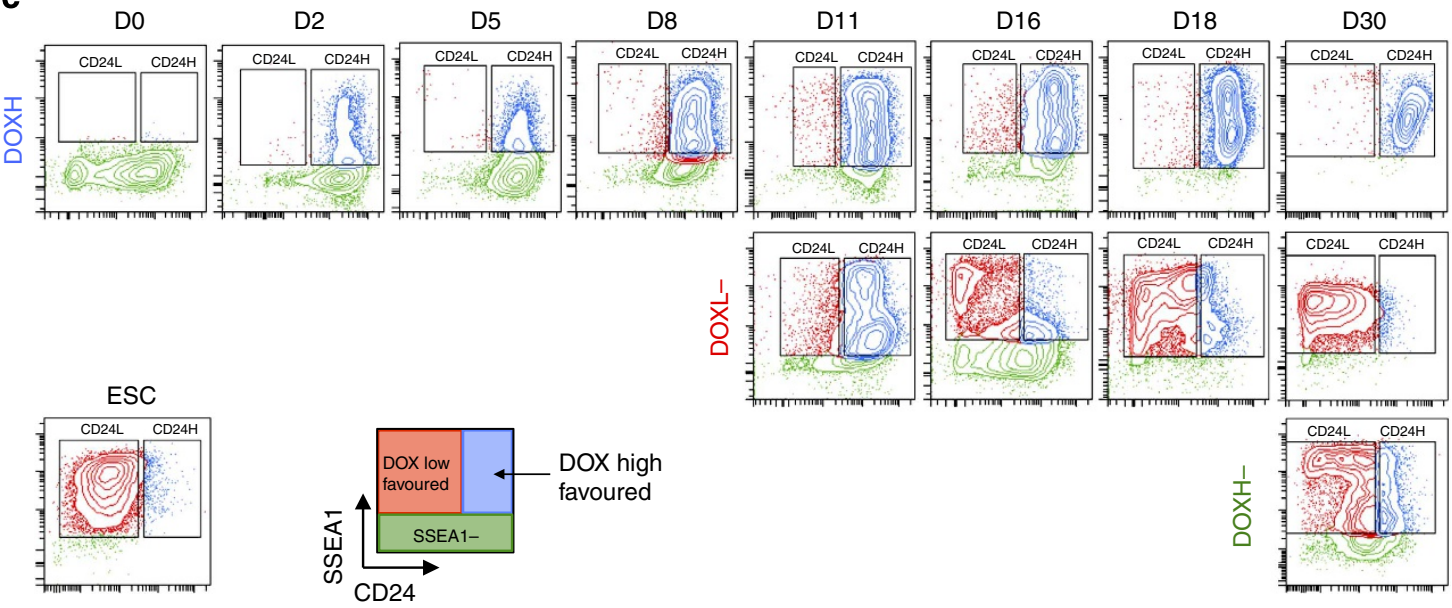

Figure 1 | Surface proteome analysis during reprogramming identifies CD24 as a differentially expressed surface marker. (a) Principal component analysis of the surface proteome (including only the subset of surface proteins that were also present in the global proteome screen to improve rigour of quantitative analysis) of reprogramming secondary MEF 1 B cells derived from tetraploid complementation, showing divergent routes of F-class and ESC-like iPSCs. (b) Summary of sampling time course and DOX treatment protocols. (c) Representative flow cytometry plots of CD24 versus SSEA1 expression during reprogramming of secondary MEF $1 B$ cells derived from tetraploid complementation, revealing emerging CD24high/SSEA1 + (CD24H) and CD24 ${ }^{\text {low }} /$ SSEA1 + (CD24L) subpopulations. ESC control is included for comparison. Flow plots are representative from three biological replicates.

MEF states. This produced a list of genes, of which many were metabolic or neural markers. A handful of proteins were chosen based on this analysis as well as antibody availability (Supplementary Fig. 3) for validation along the reprogramming time course (Fig. 1b), which revealed CD24 as the best candidate based on its ability to differentiate between emerging F-class and ESC-like iPSCs (Fig. 1c). We next proceeded with a detailed characterization of CD24 expression during reprogramming.

CD24 as a marker of divergent reprogramming states. Project Grandiose 'omic' data show transcriptional upregulation of CD24 expression following DOX addition and a reduction in the ESClike iPSC state (Supplementary Fig. 4a). Furthermore, the CD24 locus exhibits high levels of $\mathrm{H} 3 \mathrm{~K} 4 \mathrm{me} 3$ and $\mathrm{H} 3 \mathrm{~K} 36 \mathrm{me} 3$ activation marks during the reprogramming time course, with elevated $\mathrm{H} 3 \mathrm{~K} 27 \mathrm{me} 3$ repressive mark at the final ESC-like iPSC stage ${ }^{10}$ (Supplementary Fig. 4a). This trend was conserved in previously published reprogramming systems ${ }^{3,4,8,19,20}$, where the levels of CD24 increase following reprogramming induction and downregulate at the iPSC state where ectopic transgene expression is removed (Supplementary Fig. 5). Furthermore, CD24 levels are further reduced in the Thy1 + fraction of cells as compared with the reprogramming Thyl-/SSEA1 + fractions in the Polo et al. data set, supporting the view that expression of
CD24 can help distinguish reprogramming cells from somatic and pluripotent cell states (Supplementary Fig. 5d).

Recently, CD44 and Icam1 were used to track the progression of reprogramming MEFs as they move through CD44+1 Icam $1-$ and CD $44-/$ Icam $1-/ \mathrm{Nanog}+$ states to reach a final CD44-/Icam1 + iPSC state ${ }^{6}$. CD44/Icam1 dynamics in the Project Grandiose data exhibited a similar trajectory, with CD44 transcriptome levels reaching maximal levels following DOX addition and decreasing as the ESC-like iPSC state is reached, consistent with the acquisition of the $\mathrm{H} 3 \mathrm{~K} 27 \mathrm{me} 3$ repressive mark at the ESC-like iPSC state ${ }^{10}$ (Supplementary Fig. 6a). Icam1 levels decrease following DOX removal and increase as cells progress through reprogramming, reaching a maximal level at the ESC-like iPSC state where $\mathrm{H} 3 \mathrm{~K} 27 \mathrm{me} 3$ repression marks are lost and $\mathrm{H} 3 \mathrm{~K} 36 \mathrm{me} 3$ activation marks are gained ${ }^{10}$ (Supplementary Fig. 6a). In order to assess the utility of combining CD44/Icam1 and CD24 as markers to delineate divergent reprogramming populations, we next evaluated the expression of these markers on live reprogramming cells using flow cytometry.

Analysis of CD24 expression was conducted using $2^{\circ} \mathrm{MEFs}$ treated in the DOX-high (DOXH), DOX-low-to-negative $(\mathrm{DOXL}-)$ and DOX-high-to-negative (DOXH - ) time course, as previously described (Fig. 1b) ${ }^{10}$. Importantly, CD24 expression levels showed concordance across flow cytometry and mass spectrometry platforms (Supplementary Fig. 4b). Flow cytometry 
for CD24/SSEA1 expression along the three DOX time courses revealed the emergence of a $\mathrm{CD} 24^{\text {high }} / \mathrm{SSEA} 1+$ population in the DOXH condition, hereafter referred to as CD24H cells, while a $\mathrm{CD} 24^{\text {low }} / \mathrm{SSEA} 1+$ population stabilized in the DOXL - and $\mathrm{DOXH}$ - conditions, hereafter referred to as CD24L cells (Fig. 1c, Supplementary Fig. 7a). The gating strategy henceforth used to define and quantify $\mathrm{CD} 24 \mathrm{H} / \mathrm{L}$ cells is shown in Supplementary Fig. 7b. Importantly, when the CD24/SSEA1 staining strategy was applied to a different reprogramming system, Collal secondary reprogramming $\mathrm{MEFs}^{21}$, the utility of CD24 as a marker for tracking reprogramming is conserved (Supplementary Fig. 8a). As anticipated, DOX treatment upregulated CD24 such that nearly all cells $(93.8 \pm 0.4 \%)$ were CD24 high by 2 days (Supplementary Fig. 8a). While this reprogramming system did not give rise to SSEA1 + cells as quickly as the $1 \mathrm{~B}$ system, a small $\mathrm{CD} 24 \mathrm{H}$ fraction emerged after 8 days of DOX treatment (Supplementary Fig. 8a); however, this $\mathrm{CD} 24 \mathrm{H}$ subpopulation was largely transient and the CD24L fraction dominated (Supplementary Fig. 8a). This is consistent with the observation of Tonge et al. ${ }^{22}$ that these cells do not stabilize in an F-class state.

F-class cells are known to homogenously emerge in $\mathrm{DOXH}$ conditions (using the $2^{\circ} \mathrm{MEF} 1 \mathrm{~B}$ tetraploid system) while ESClike iPSCs are observed solely in DOX-negative (DOX -) conditions ${ }^{9,10}$. This suggested that $\mathrm{CD} 24 \mathrm{H}$ cells (in DOXH) may correspond to F-class cells, while CD24L cells may correspond to ESC-like iPSCs. Control ESCs exhibited a CD24L staining profile, supporting this hypothesis (Fig. 1c). F-class cells derived from primary DOX-inducible $1 \mathrm{~B}$ cells as well as tail tip fibroblasts also showed a consistent $\mathrm{CD} 24 \mathrm{H}$ expression profile (Supplementary Fig. 8b). Interestingly, secondary MEFs derived from a $27 \%$ chimera, exhibiting different OKMS expression levels, also traverse a $\mathrm{CD} 24 \mathrm{H}$ state following DOX induction (Supplementary Fig. 9). This observation also supports the fact that OKMS stoichiometry plays a role in directing cell fate in reprogramming ${ }^{9,10}$. In fact, a recent report shows that lower levels of Klf4 overexpression during reprogramming stalls the cells in a partially reprogrammed, transgene-dependent state ${ }^{23}$, which is consistent with our observation that, while MEF 1Bs derived from tetraploid complementation give rise to F-class cells robustly, 1B MEFs from $27 \%$ chimeras exhibit higher levels of Klf4 transgene (Supplementary Fig. 9c) and are able to give rise to ESC-like iPSCs in DOXH culture (Supplementary Fig. 9a).

While CD24 shows potential for demarcating divergent populations emerging from unique DOX treatment time courses, flow cytometry staining for CD44/Icam1 shows DOXH-treated cells prematurely transition to a CD44-/Icam1 + state, which is reportedly indicative of Nanog + iPSCs $^{6}$ (Supplementary Fig. 6b,c). Thus, CD44/Icam1 cannot demarcate F-class and ESClike iPSCs, demonstrating the utility of CD24 for categorizing reprogramming populations. Interestingly, CD $44+/$ Icam $1+$ cells maintain the highest proportions of $\mathrm{CD} 24^{\text {high }}$ cells, while CD44-/Icam1 + cells exhibit the lowest proportion of CD24high cells in intermediate reprogramming stages (Supplementary Fig. 6d). As a positive marker for the F-class state, CD24 allows for a direct demarcation of divergent reprogramming from an ESC-like state, which is well characterized and identified by surface proteins such as Pecam1, E-cadherin ${ }^{3}$ and CD44/Icam1 (ref. 6).

Characterization of $\mathrm{CD} 24 \mathrm{H}$ and $\mathrm{CD} 24 \mathrm{~L}$ subpopulations. In order to comprehensively compare CD24H and CD24L cells to F-class/ESC-like iPSCs, we chose the following criteria to characterize the newly defined cells (Fig. 2a): CD24/SSEA1 expression levels compared with ESC controls; morphology in native culture; morphology in response to DOX removal; stability following extensive passaging; dependence of proliferation on DOX; and gene expression profile.

Quantification of the percentage of CD24H and CD24L cells emerging in the DOX time courses suggested that $\mathrm{CD} 24 \mathrm{H}$ cells arise homogenously in DOXH culture, exhibiting an F-class morphology, while CD24L cells emerge in DOXL - and DOXH - culture, exhibiting an ESC-like morphology (Fig. 2b,c, Supplementary Fig. 10a). Control ESCs expressed a CD24 low $/$ SSEA $1+$ profile, consistent with CD24L cells (Figs 1c and $2 \mathrm{~b}$ ). This trend was independent of passaging frequency (Supplementary Fig. 10b). Following sorting for the CD24H and CD24L populations from DOXH and DOXL - time courses on day 30 of reprogramming, $\mathrm{CD} 24 \mathrm{H}$ cells maintained their F-class morphology while DOX removal resulted in the loss of these cells (Fig. 2d), consistent with reports from Tonge et al. ${ }^{9}$ On the other hand, CD24L-sorted cells maintained their ESC-like morphology and were stable in both DOXH and DOX - conditions (Fig. 2d).

Continued passaging of CD24H cells in DOXH and CD24L cells (from DOXL - and DOXH - time courses) in DOX conditions revealed that these cells maintained their CD24 expression profiles in their native DOX conditions (Fig. 2e; Supplementary Figs 10c and 11a). Consistent with the observation of CD24H DOX dependence, removal of DOX in these cells resulted in reduced proliferation, assessed by 5-ethynyl- $2^{\prime}$ deoxyuridine (EdU) staining (Fig. 2f). Gene expression analysis showed $\mathrm{CD} 24 \mathrm{H}$ cells (derived from day $30 \mathrm{DOXH}$ culture) clustered with F-class cells (taken from Tonge et al. ${ }^{9}$ ), while CD24L cells (derived from day $30 \mathrm{DOXL}$ - and DOXH culture) clustered with ESCs, confirming the ability of CD24/ SSEA1 staining to demarcate F-class and ESC-like iPSC populations (Fig. 2g). As expected, ESC-like iPSC markers, Pecam1 and E-cadherin, were seen in CD24L cells while not in CD24H cells (Supplementary Fig. 11b). Furthermore, CD24L cells exhibited levels of OKMS expression that are consistent with ESCs (Fig. 2g). Consistent with Tonge et al. ${ }^{9}$, both CD24H and CD24L cells were able to contribute to the three germ layers following in vitro differentiation (Supplementary Fig. 12). Overall, these studies reveal that $\mathrm{CD} 24$ can separate the transgene-dependent F-class iPSCs from the transgene-independent ESC-like iPSCs.

CD24 demarcates transgene-independent pluripotent states. We have shown that CD24 can be used to demarcate transgenedependent F-class cells from ESC-like iPSCs. Given the role of CD24 in separating these iPSC states, we wondered whether CD24 would show conservation and utility in nonreprogrammed pluripotent populations. In order to investigate this possibility, we costained both mouse ESC and epiblast stem cell (EpiSC) populations for CD40 (a known EpiSC surface marker ${ }^{24}$ ) and CD24. Staining for CD24/CD40/SSEA1 levels in control mouse ESCs and EpiSCs confirmed the ability of CD24 $4^{\text {high }} / \mathrm{CD} 40+$ staining to identify the EpiSC state, while ESCs were CD24 ${ }^{\text {low }} / \mathrm{CD} 40-$ (Fig. 3a). In order to better characterize the rare CD24 ${ }^{\text {high }}$ cells emerging in standard ESC populations, we cultured ESCs in serum and LIF conditions and sorted for the rare CD24 $4^{\text {high }} / \mathrm{CD} 40+$ (EpiSC-like) as well as the prevalent CD24 ${ }^{\text {low }} /$ SSEA $1+$ (standard ESC) fraction of cells and conducted a survey of pluripotency and early differentiation gene expression ${ }^{25}$ (Fig. 3b). As expected, EpiSC-like CD24 $4^{\text {high} / C D 40 ~+~ c e l l s ~ e x h i b i t e d ~ l o w e r e d ~ l e v e l s ~ o f ~}$ naive pluripotency genes such as Stella, Rex1 and Nanog while also exhibiting higher levels of Foxa2, Eomes, Gata6, Sox17, Cer1 and Fgf5 (Fig. 3b). The observation of this rare EpiSC-like fraction of cells in serum-based ESC culture has been previously observed ${ }^{26,27}$.

Importantly, CD40 staining was negative in F-class and ESClike iPSCs derived from the DOXH or DOXH - reprogramming 


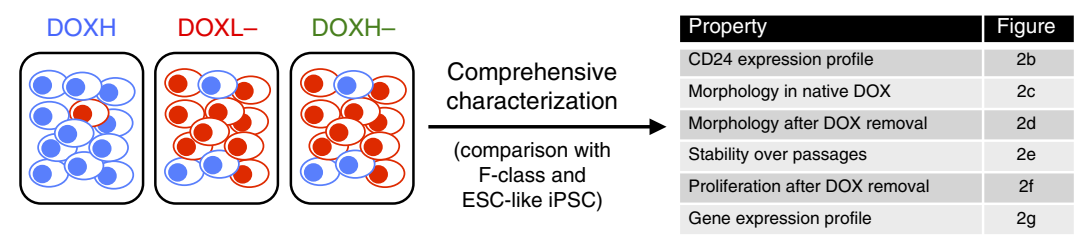

b
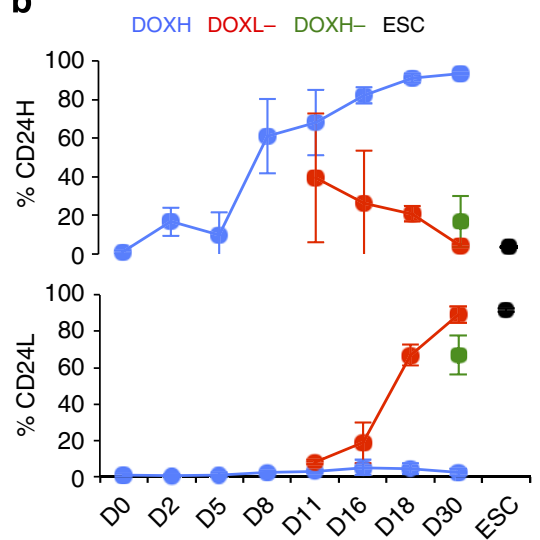

e

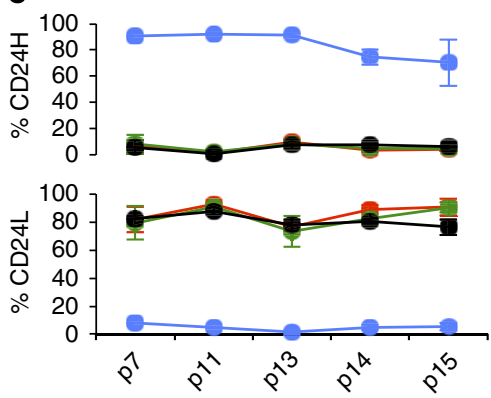

C
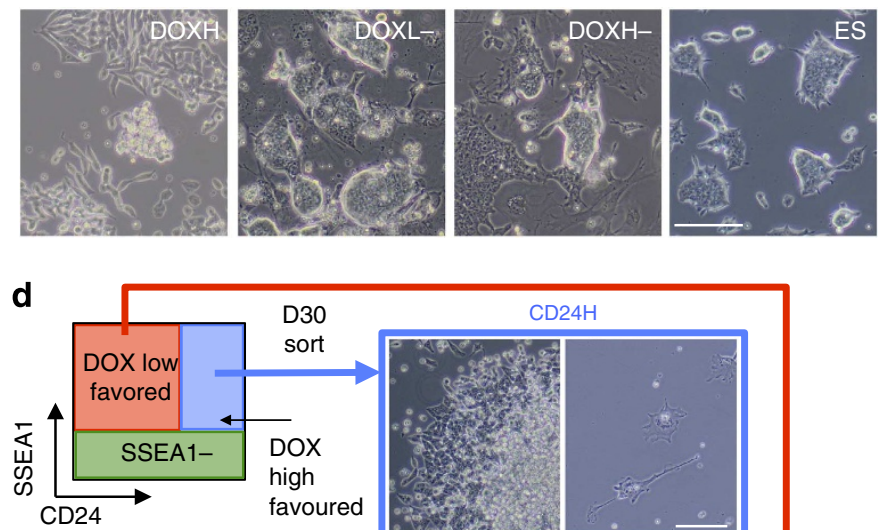

f

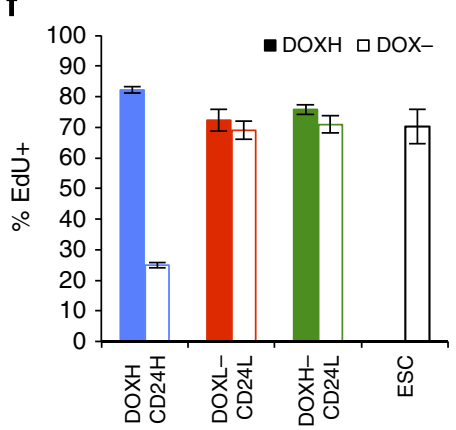

$\mathrm{DOXH}$

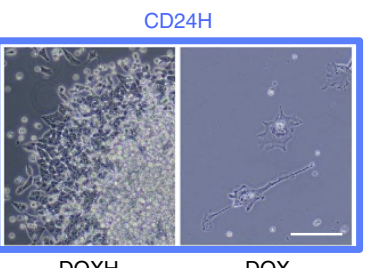

DOX-

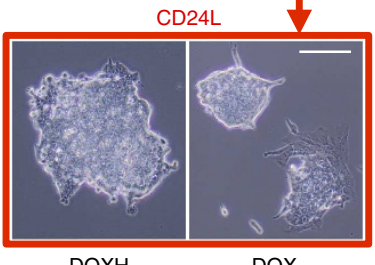

DOXH

DOX-

DOXH CD24H cells in DOXH

DOXL-CD24L cells in DOXDOXH- CD24L cells in DOXESC

g

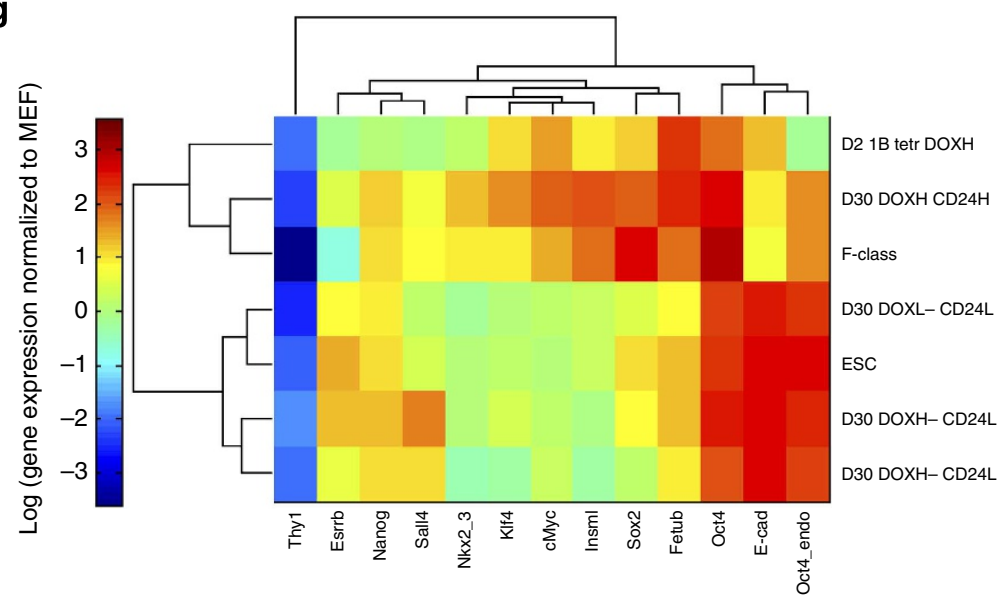

Figure 2 | CD24H and CD24L subpopulations correspond to transgene-dependent (F-class) and ESC-like iPSCs, respectively. (a) Summary of approach to characterize CD24H and CD24L populations. (b) Percentage of CD24H and CD24L cells in DOXH, DOXL - and DOXH - culture time courses. Data bars show mean \pm s.d. ( $n=3$ biological replicates). (c) Representative phase contrast images ( $n=3$ biological replicates) of emerging colonies in the three DOX treatments, including ESC control. Scale bar, $125 \mu \mathrm{m}$. (d) Summary of sorting strategy used to separate CD24H and CD24L subpopulations from D30 culture. Representative phase contrast images ( $n=3$ technical replicates) of sorted CD24H and CD24L cells in DOXH and DOX - conditions. Scale bar, $125 \mu \mathrm{m}$. (e) Effect of long-term passaging on levels of CD24H and CD24L cells in D30-sorted DOXH CD24H, DOXL - CD24L and DOXH - CD24L cells. ESCs are included as a control. Data bars show mean \pm s.d. ( $n=3$ technical replicates). (f) EdU staining of DOXH CD24H, DOXL - CD24L and DOXH CD24L cells in DOXH and DOX - conditions to assess DOX dependence of proliferation. Data bars show mean \pm s.d. ( $n=3$ technical replicates).

(g) Expression levels of various pluripotent and F-class-specific genes in CD24H and CD24L cells with unsupervised hierarchical clustering, including F-class and ESC cell controls, normalized to Gapdh and MEF cells. 

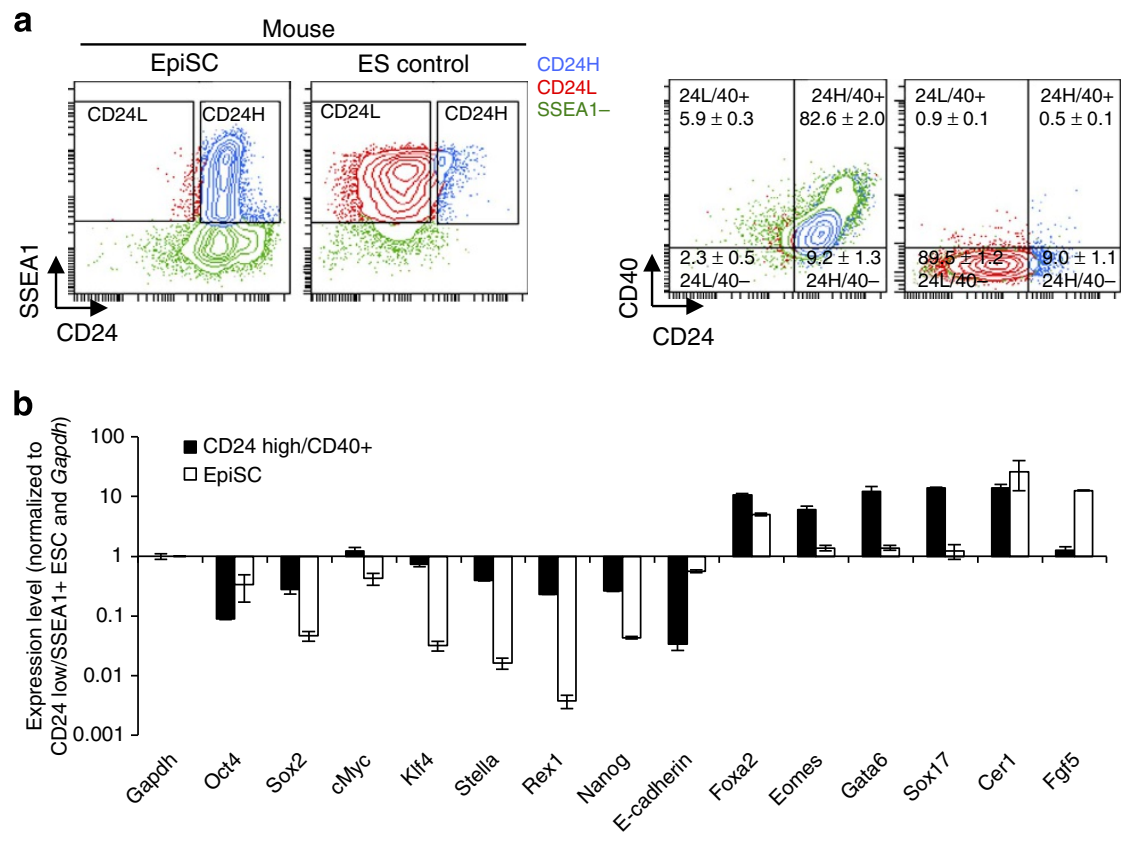

Figure 3 | CD24 and CD40 exhibit differential expression on mouse ESCs and EpiSCs. (a) Flow cytometry analysis of CD24/SSEA1/CD40 expression in mouse ESCs and EpiSCs. Representative flow plots ( $n=3$ technical replicates) of stained mouse ESC and control embryo-derived EpiSCs. (b) Gene expression of CD24high $/ C D 40+$ cells derived from R1 ESC culture compared with control embryo-derived EpiSCs. The gene panel is composed of pluripotency genes as well as some early differentiation markers. Values are normalized to sorted CD24low/SSEA1+ (ESC-like) cells sorted from R1 ESC culture and Gapdh. Data bars show mean \pm s.d. ( $n=3$ technical replicates).

time courses, while some levels of CD40 expression was evident in the cells derived from the DOXL - time course (Supplementary Fig. 13).

Finally, we tested whether culture-derived EpiSCs, generated from transgene-independent iPSCs selected from our 1B reprogramming cultures as well as an ESC control, would acquire the predicted $\mathrm{CD} 24^{\text {high }} / \mathrm{CD} 40+$ state. Importantly, all iPSC replicates as well as the ESC control were able to give rise to CD24 high/CD40 + cells with an EpiSC-like morphology and gene expression profile, while $2 \mathrm{i}$ treatment abolished any rare $\mathrm{CD} 24^{\text {high }} / \mathrm{CD} 40+$ cells that remain in serum-based culture over continued passages (Supplementary Fig. 14). Together, these data support the notion that $\mathrm{CD} 24^{\text {high }}$ cells in transgene-free mouse pluripotent culture represent a rare fraction of EpiSC-like cells.

CD24 expression in human PSCs. Given the hypothesized similarities between mouse EpiSCs and human ESCs, we wondered whether CD24 expression is conserved on human (h) pluripotent stem cells (PSCs) ${ }^{24}$. Analysis demonstrated that CD24 expression is conserved in hESCs. Thus, CD24 serves as a tool to capture the transition from low CD24 expression in starting cells to high CD24 expression following OKMS induction and movement to a human iPSC state 28,29 , exhibiting levels of CD24 that are comparable with hESCs and are higher than the starting somatic cells (Supplementary Fig. 15a,b). This is consistent with one hypothesis that considers hESCs as 'primed', analogous to the mouse EpiSC state, and in contrast to the 'naive' cell state of mouse $\mathrm{ESCs}^{30}$. Data mining for expression of CD24 in the developing human embryo with single-cell RNA-seq resolution ${ }^{31}$ revealed increasing CD24 levels as the zygote reached a post-implantation (primed) state with very low expression in the earliest (naive) embryonic states (Supplementary Fig. 15c).

In order to test this in vitro we cultured hESC lines in two conditions: standard hESC culture and culture supplemented with conditions reported to induce hESCs to an alternative naivelike state ${ }^{11}$. Flow cytometry analysis of these cells confirmed that CD24 expression is lowered in naive-like hESC states, achieving levels equivalent with mouse ESCs by passage 10 of culture in so-called naive conditions ${ }^{11}$ (Fig. 4a). Further development of our naive induction strategy led us to note a dependency of CD24 downregulation on levels of $\mathrm{LDN}$ in our naive induction protocol (Supplementary Fig. 16a). To ensure that CD24 was indeed a reliable marker for the primed-to-naive-like state transition, we removed LDN from subsequent experiments. hESCs were costained for CD24/Tra-1-60 (analogous to mouse CD24/SSEA1 staining) following changes in primed-to-naive associated culture conditions (Fig. 4b). As before, an observable (although reduced in size) CD24 ${ }^{\text {low }} /$ Tra-1-60 + fraction emerged. Analysis of Oct4/Sox2 expression in naive-like cells revealed maintenance of pluripotency (Supplementary Fig. 16b). Our observation was further supported by CD24 expression data extracted from a recent study by Theunissen et al. ${ }^{32}$, reporting generation of naive hESCs without LDN (Supplementary Fig. 15d). Characterization of our naive hESCs revealed that they exhibited gene expression levels consistent with previous reports ${ }^{11,32,33}$. Furthermore, gene expression analysis revealed that sorted CD24 $4^{\text {high }} /$ Tra- $1-60+$ (hereafter called $\mathrm{CD} 24 \mathrm{H})$ cells and CD24low/Tra-1-60+ (hereafter called CD24L) cells clustered distinctly (Fig. 4c). Interestingly, $\mathrm{CD} 24 \mathrm{H}$ cells derived from primed and naive-treated cultures clustered together, exhibiting especially low levels of Tbx3 expression (Fig. 4c,d) and expectedly higher levels of CD24a expression (Supplementary Fig. 16c). In addition, these CD $24 \mathrm{H}$ cells clustered with the other primed cells. On the other hand, CD24L cells from naive culture clustered with unsorted naivetreated cells. Most notably, while unsorted naive hESCs derived here showed enrichment for naive markers Klf2, Tbx3, Otx2, Dnmt3a, LIF-R and Rex1, CD24L-sorted naive cells showed further enrichment for naive markers Stella, E-cadherin, Klf5 and Klf4 (Fig. 4d). This analysis may suggest heterogeneity in these naive hESC cultures, and support CD24 as a powerful tool to 
a

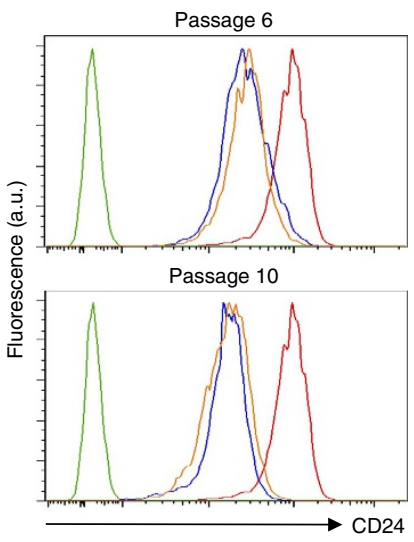

Unstained

'Primed' hESe $\square$ 'Naiv

C

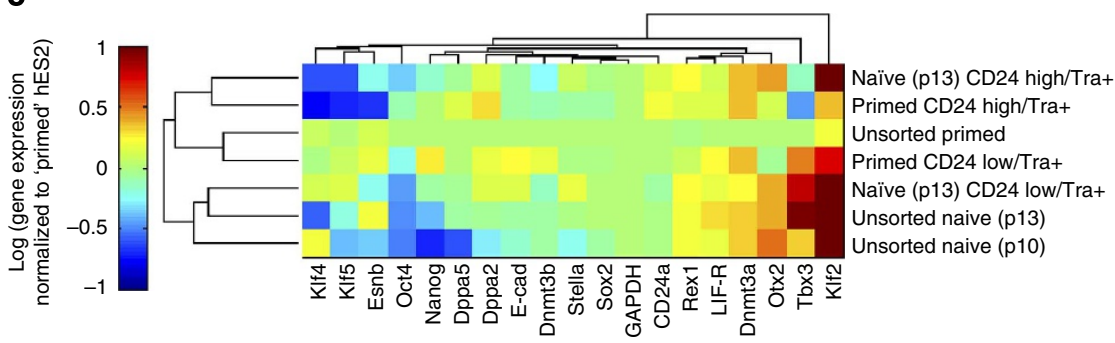

d

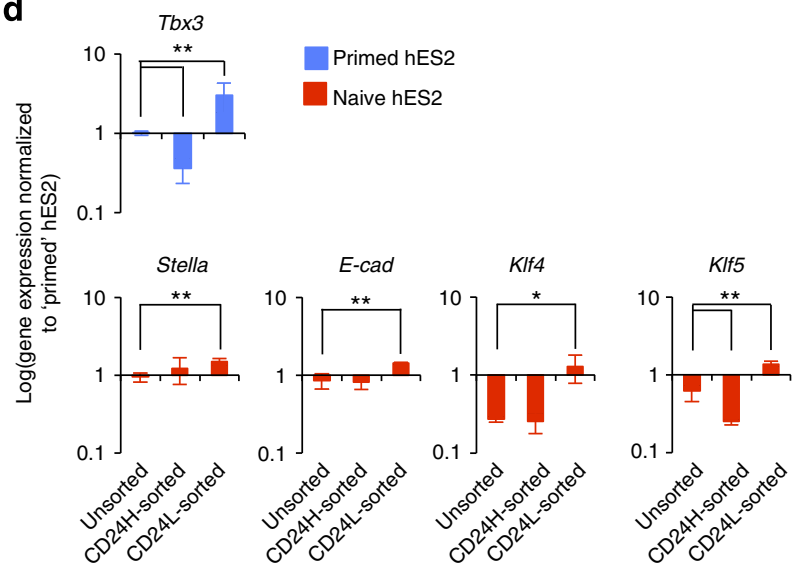

b
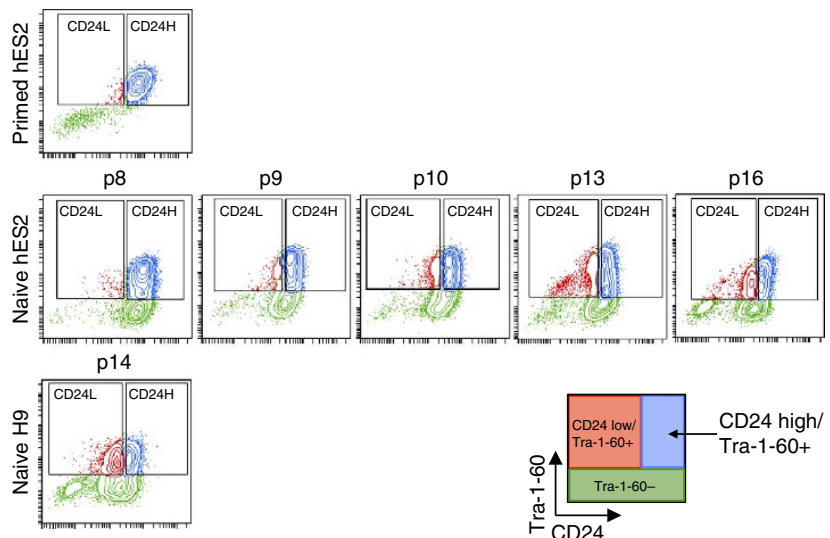

'Primed' hES2 $\square$ 'Naïve' H9 
ectoderm, mesoderm and ventral endoderm ${ }^{39}$. In general, CD24 is expressed at higher levels in progenitor cells and metabolically active cells than in terminally differentiated cells ${ }^{38}$. CD24 has been shown to support cell adhesion of myeloid cells to P-selectin, whereby P-selectin acts as a ligand, triggering downstream src-family tyrosine kinases ${ }^{34}$. In the cancer literature, CD24 has been found to correlate with aggressive tumour behaviour ${ }^{40,41}$ and serves as a marker for diagnosis and prognosis ${ }^{38}$. For example, CD24+ hepatocellular carcinoma cells showed an increased propensity for self-renewal, differentiation and metastasis as well as enriched levels of Sox 2 and Oct4 expression $^{40}$. Importantly, knockdown of CD24 suppressed these characteristics, supporting the functional role of CD24 in tumorigenesis. Nanog has also been identified as an important downstream effector of CD24-mediated tumorigenicity and selfrenewal, where CD24 phosphorylates STAT3 through $\mathrm{src}^{40}$. CD24-mediated c-src kinase activity has also been reported to promote integrin-mediated adhesion, epithelial-to-mesenchymal transition and invasion in breast cancer $^{42,43}$. Furthermore, a recent report has shown that $\mathrm{CD} 24$ affects cell cycle dynamics, whereby CD24 plays a role in functionally inactivating p53 in human prostate cancer cells ${ }^{44}$. These reported signalling cascades may provide insight into the $\mathrm{CD} 24$ status in pluripotent culture as well, as there has been clear links between CD24 and core pluripotency and cell cycle regulators.

The cell surface proteome provides a means of accessing the cell state without sacrificing viability ${ }^{6}$. We used mass spectrometry to measure changes in surface glycoprotein ${ }^{12}$ expression throughout the reprogramming time course and found that cell surface proteins are markedly over-represented in proteins undergoing two- or higher fold changes in expression. This suggested that the surface proteome serves as a rich data set to identify markers that track cell state changes during reprogramming.

CD24 is upregulated quickly after reprogramming induction, reaching maximal levels within a few days of transgene overexpression in both the 1B and Collal systems, with similar trends observed in other reported reprogramming systems. As a result, $\mathrm{CD} 24$ acts as a good marker to track the initiation of reprogramming following OKMS overexpression, during which the cells acquire a CD24high/SSEA1 - state. In the $1 \mathrm{~B}$ system, cells traversing the reprogramming route can become stabilized in a $\mathrm{CD} 24^{\text {high }} / \mathrm{SSEA} 1+$ state, which is shown here to correspond to recently discovery transgene-dependent F-class iPSCs, derived from high transgene reprogramming ${ }^{9}$. Cells that are able to acquire a transgene-independent ESC-like iPSC state are characterized by
CD2 $4^{\text {low }} / \mathrm{SSEA} 1+$ expression. Following transgene removal, CD24 also shows the ability to distinguish primed and naive transgene-independent pluripotent states. Published RNA-Seq data comparing mouse ESC and EpiSC states suggest that EpiSCs exhibit higher CD24 levels ${ }^{45}$. Here we have confirmed that CD24 shows strong correlation with the known EpiSC marker, CD40 (ref. 24), and is able to separate ESCs and EpiSCs. Thus, we have shown that three observed states in pluripotent culture could be identified as a function of culture conditions and CD24 expression in the following manner: $\mathrm{CD} 24 \mathrm{H}$ cells in DOXH culture, which are transgene-dependent, F-class, iPSCs; CD24L cells in DOX -, which are ESC-like iPSCs; and CD24high cells in DOX -, which are EpiSCs. The dual role of $\mathrm{CD} 24$ in delineating these three populations is outlined in Fig. 5b. An important methodological advantage of CD24 during reprogramming is its ability to replace the use of reprogramming state reporters. Other end-stage iPSC surface markers, such as Pecam1 and E-cadherin, have the added difficulty of being sensitive to trypsinization ${ }^{46,47}$ and therefore are not ideal for use. Furthermore, CD24 allows for the identification of cells diverting to a transgene-dependent iPSC state, and the separation of this cell type from more ESC-like iPSCs.

Importantly, CD24 also shows conservation in the human system. Consistent with the hypothesis that hESCs are in a primed pluripotent state $\mathrm{e}^{30}$, they exhibit high levels of CD24, analogous to mouse EpiSCs. Thus, CD24 may provide some utility in tracking the conversion towards a human iPSC state, which is characterized by high CD24 levels ${ }^{28,29}$. The recent identification of culture conditions that promote conversion to naive-like hESCs ${ }^{11,48}$ enabled the demonstration that CD24 tracks these changes in the human system. Interestingly, during in vivo human embryonic development, CD24 levels increase at the single-cell level as the blastocyst undergoes implantation and reaches an epiblast stage ${ }^{31}$. Furthermore, naive-induced hESC populations that were sorted for $\mathrm{CD} 24^{\text {low }}$ cells were further enriched for naive hESC gene expression. Thus, CD24 serves as a marker for resolving heterogeneity in the naive hESC pool.

\section{Methods}

Identification of cell surface proteins. A streamlined version of the cell surface capture protocol introduced in ref. 12 with simplified sample handling was applied to identify $\mathrm{N}$-glycosylated surface proteins over the project time course. The steps involved are described in detail here. Between $5 \times 10^{7}$ and $30 \times 10^{7}$ cells (individual counts varied by time points) were collected in a 50 -ml tube and pelleted through centrifugation $(350 \mathrm{~g})$, and then placed on ice. The pellets were resuspended once in ice-cold labelling buffer (PBS-adjusted to $\mathrm{pH} 6.5$ with $85 \%$ phosphoric acid), and then again pelleted through centrifugation. a
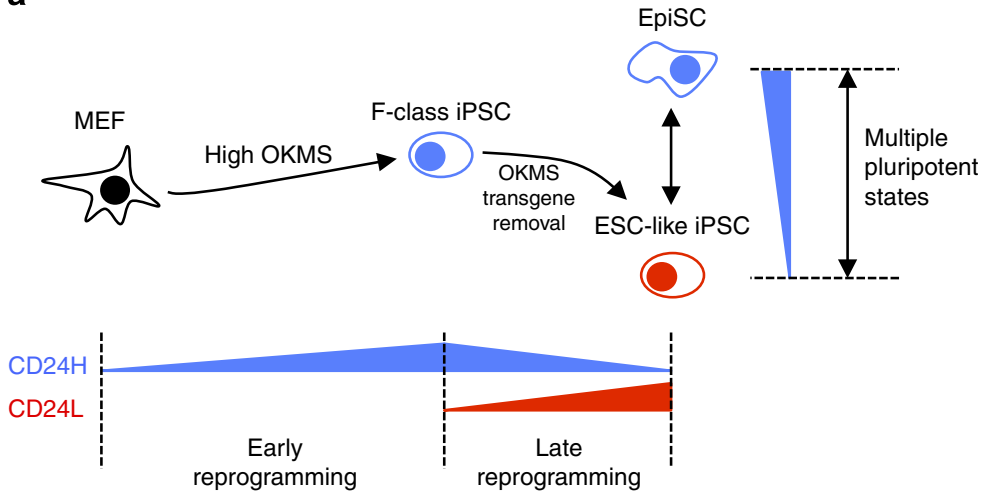

b

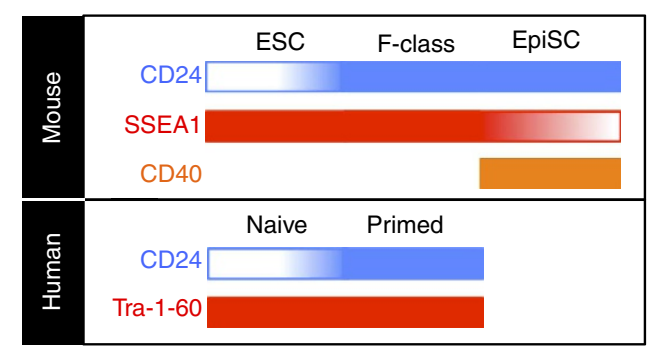

Figure 5 | Summary of CD24 as a marker for three pluripotent states. (a) Overview of CD24 as a tool for tracking transgene induction in early reprogramming, demarcating divergent reprogramming to F-class and ESC-like iPSC states in late reprogramming, and identifying multiple pluripotent states in transgene-independent pluripotent culture. (b) Summary of approach for identifying mouse F-class iPSCs, ESC-like iPSCs and EpiSCs via CD24/ SSEA1/CD40 staining as well as human primed and naive hESCS via CD24/Tra-1-60 staining. 
The cells were subsequently resuspended and oxidized for $15 \mathrm{~min}$ in the dark at $4{ }^{\circ} \mathrm{C}$ with $1.6 \mathrm{mM}$ sodium-meta-periodate (Sigma) in labelling buffer while being gently agitated. The cell pellet was washed once with labelling buffer to remove residual sodium-meta-periodate and to deplete dead cells and cell fragments. Following centrifugation, the cell pellet was resuspended and the cells exposed to the labelling agent, biocytin hydrazide (Biotium), $5 \mathrm{mM}$ in $10 \mathrm{ml}$ labelling buffer, for $60 \mathrm{~min}$ at $4{ }^{\circ} \mathrm{C}$ on a rotator on slow speed. On labelling, the cell pellet was washed once with ice-cold labelling buffer to remove unreacted biocytin hydrazide and to deplete dead cells/fragments.

The cell pellet was resuspended in $1 \mathrm{ml}$ of ice-cold $0.1 \mathrm{M}$ ammonium bicarbonate (Sigma) and then transferred to a 1.5-ml Eppendorf tube. After $30 \mathrm{~s}$ of indirection sonication on a VialTweeter (Hielscher) at $100 \%$ amplitude and 0.5 cycle time, the suspension was centrifuged at $400 \mathrm{~g}$ at $4{ }^{\circ} \mathrm{C}$ for $2 \mathrm{~min}$ and the size of the (now reduced) pellet noted. Additional sessions of $15 \mathrm{~s}$ of sonication followed by centrifugation were performed until the pellets no longer appeared to change in size. The lysate was then centrifuged at $2,500 \mathrm{~g}$ at $4{ }^{\circ} \mathrm{C}$ for $15 \mathrm{~min}$. The still-cloudy supernatant was transferred to another Eppendorf tube. After thorough mixing, two 21 aliquots were set aside to perform a duplicate DC protein assay (Bio-Rad). The remaining supernatant was stored at $-80^{\circ} \mathrm{C}$.

The supernatant was thawed on ice once needed. A proportion calculated to hold $5 \mathrm{mg}$ of protein was transferred to a separate tube and the remainder put back in storage. Sufficient $100 \mathrm{mM}$ ammonium bicarbonate was added to bring the volume up to $1 \mathrm{ml}$, following which $100 \mathrm{ml}$ of $1 \%$ RapiGest (Waters) in $100 \mathrm{mM}$ ammonium bicarbonate was added to bring the final concentration to $0.1 \%$. This membrane preparation was indirectly sonicated at $100 \%$ amplitude and 0.5 cycle time for $3 \mathrm{~min}$ in a VialTweeter to obtain a translucent solution. Reduction: $100 \mathrm{mM}$ TCEP (Sigma) in $100 \mathrm{mM}$ ammonium bicarbonate was added to a final concentration of $5 \mathrm{mM}$ TCEP. After $3 \mathrm{~min}$ as before in a VialTweeter, the sample was allowed to stand for $30 \mathrm{~min}$ at room temperature. Alkylation: $500 \mathrm{mM}$ iodoacetamide (Sigma) in $100 \mathrm{mM}$ ammonium bicarbonate was added to a final concentration of $10 \mathrm{mM}$ iodoacetamide and the sample incubated for $30 \mathrm{~min}$ in the dark at room temperature. Digestion: $50 \mathrm{mg}$ of trypsin (Promega) was added to establish a 1:100 ratio with the measured protein content. The proteins were digested overnight at $37^{\circ} \mathrm{C}$ while the sample was gently tumbled. After digestion, the peptide mixture was centrifuged for $10 \mathrm{~min}$ at $15,000 \mathrm{~g}$ and the supernatant transferred to a new Eppendorf tube, which was then heated for $15 \mathrm{~min}$ at $95^{\circ} \mathrm{C}$ to inactivate the trypsin.

UltraLink Streptavidin Plus bead slurry $(200 \mathrm{ml}$; Piercenet) was placed in a Mobicol (Bocascientific) and washed four times with $100 \mathrm{mM}$ ammonium bicarbonate. The beads were resuspended in $100 \mathrm{mM}$ ammonium bicarbonate and added to the peptide mixture. Together, they were incubated for $1 \mathrm{~h}$ at room temperature to allow the labelled peptides to bind to the beads. The beads were then returned to a Mobicol and washed intensively: 20 times with $5 \mathrm{M} \mathrm{NaCl}, 20$ times with Stim-Lys buffer, and then finally 20 times with $100 \mathrm{mM}$ ammonium bicarbonate heated to $60^{\circ} \mathrm{C}$. The Stim-Lys buffer consisted of $137 \mathrm{mM} \mathrm{NaCl}$, $50 \mathrm{mM}$ Tris adjusted to $\mathrm{pH} 7.8$ with $\mathrm{HCl}, 100 \mathrm{mM}$ glycerol, $0.5 \mathrm{mM}$ EDTA adjusted to $\mathrm{pH} 8.0$ with $\mathrm{HCl}$ and $1 \%$ Triton X (all Sigma). Washing was performed by immersing beads while in the Mobicol, and then spinning out the liquid content by centrifuging for $2 \mathrm{~min}$ at $2,000 \mathrm{~g}$.

The beads were again immersed, in $400 \mathrm{ml}$ of $100 \mathrm{mM}$ ammonium bicarbonate (still within the Mobicol), to which $1 \mathrm{ml}$ of PNGaseF (NEB) was added. They were then gently tumbled overnight at $37^{\circ} \mathrm{C}$. After incubation, the Mobicol was centrifuged for $2 \mathrm{~min}$ at 2,000 $\mathrm{g}$ and the eluate collected in an Eppendorf tube. Overall, $500 \mathrm{ml}$ of $100 \mathrm{mM}$ of ammonium bicarbonate in $4 \%$ acetonitrile was added to resuspend the beads and was collected in the same manner. The two eluates were combined and then acidified with $150 \mathrm{ml}$ of $10 \%$ formic acid to below $\mathrm{pH} 3.0$. This glycopeptide mixture was vortexed until no more bubble formation was apparent, and then stored at $-80^{\circ} \mathrm{C}$ for later liquid chromatography-mass spectrometry (LC-MS).

Microcolumns were created from capillary-scale nanoflow 75- $\mu \mathrm{m}$ I.D. fused silica tubing (Polymicro Technologies) and then pulled to a fine tip using a P-2000 laser puller (Sutter Instruments). Each of them were packed to a length of $10 \mathrm{~cm}$ with $5 \mu \mathrm{m}$ Luna C18 resin (Phenomenix) using a pressure vessel, and then flushed for 15 min with methanol.

Chromatography buffer ' $\mathrm{A}$ ' consisted of $5 \%$ acetonitrile and $0.1 \%$ formic acid in HPLC-grade water (Fisher), while chromatography buffer 'B' consisted of $95 \%$ acetonitrile and $0.1 \%$ formic acid in HPLC-grade water.

Beginning with a proportion of the glycopeptide mixture calculated to be derived from a volume of pre-digestion sample holding $2 \mathrm{mg}$ of total protein, vacuum centrifugation was performed until the volume was concentrated to several $\mathrm{ml}$. The volume was then adjusted to $11 \mathrm{ml}$ with $0.1 \%$ formic acid and placed in a well of a 96-well plate, which in turn was placed in an EASY-nLC nano LC pump (Proxeon) connected to a microcolumn.

Microcolumns were regenerated with buffer 'A' before loading of sample by the nano LC pump. Each chromatography session began with a linear gradient elution of $5-25 \%$ buffer 'B' over $45 \mathrm{~min}$ followed by a linear gradient of $25-80 \%$ buffer 'B' over $9 \mathrm{~min}$. A flow rate of $300 \mathrm{nl} \mathrm{min}^{-1}$ was maintained. Peptides were analysed using nanospray ionization on an Orbitrap-Velos mass spectrometer (Thermo). MS and MS/MS spectra were acquired with the instrument operating in the data-dependent mode of one MS scan (on the Orbitrap) followed by up to $10 \mathrm{MS} / \mathrm{MS}$ scans (on the LTQ-Velos) when triggered by ion signals above a specified threshold. Fragmentation was accomplished using collision-induced association. Three LC-MS replicates were performed for each of the selected time points.
Database searching and analysis. All MS/MS spectra were searched against the International Protein Index mouse database (Version 3.84) using the SEQUEST algorithm. Confidences in peptide identifications were evaluated using the Statquest probabilistic mode ${ }^{49}$ and further filtered to within a mass tolerance of 20 parts-per-million using the accurate ion masses generated by the Orbitrap. This achieved an estimated false-positive rate of $2 \%$. Any identified peptides were then excluded if they did not include the $\mathrm{N}$-glycosylation consensus sequon $\mathrm{NxS} / \mathrm{T}$ or did not demonstrate the asparagine to aspartic acid deamidation of $0.986 \mathrm{Da}$ resulting from the treatment with PNGaseF. Relative quantities of cell surface proteins were assessed using spectral counting. The mass spectrometry proteomics data have been deposited to the ProteomeXchange Consortium (http://proteomecentral.proteomeexchange.org) via the PRIDE partner repository with the data set identifier PXD001456.

Culture and reprogramming of pluripotent mouse cells. MEFs (inducible $1 \mathrm{~B}$ lines ${ }^{7}$ and Collal lines ${ }^{21}$ ) and control mouse ES cells (R1 line) were maintained in Dulbecco's modified Eagle medium (DMEM) supplemented with 10\% (v/v) fetal bovine serum (FBS; Gibco), $1 \mathrm{mM}$ sodium pyruvate (Gibco), $2 \mathrm{mM}$ Glutamax (Invitrogen) and $1 \%(\mathrm{v} / \mathrm{v})$ penicillin and streptomycin (Invitrogen). Mouse fibroblasts were reprogrammed in serum-containing mouse ESC medium consisting of DMEM supplemented with 15\% (v/v) FBS (Wisent), $0.1 \mathrm{mM}$ $\beta$-mercaptoethanol (BME, Sigma), $1 \mathrm{mM}$ sodium pyruvate, $0.1 \mathrm{mM}$ MEM nonessential amino acid (NEAA, Gibco), 2 mM Glutamax, $1 \%$ (v/v) penicillinstreptomycin and 1,000 $\mathrm{U} \mathrm{ml}^{-1}$ of LIF (Millipore). EpiSCs (CDE) used for control staining were cultured as previously described ${ }^{25}$.

For reprogramming, secondary MEF $1 \mathrm{~B}$ cells (isolated as previously described ${ }^{7}$ from tetraploid complementation and chimeras) or MEF Collal cells ${ }^{21}$ were seeded as reported ${ }^{10}$, in $0.2 \%$ gelatin-coated T25 or T75 plates (Sarstedt). Cells were maintained in DOX (Sigma) high culture $\left(1.5 \mu \mathrm{g} \mathrm{ml}^{-1}\right)$ and were fed regularly and passaged at sampling time points and whenever confluence was reached. Cells were maintained on three DOX treatment time courses: DOX-high (DOXH), DOX-low-to-negative (DOXL - ) and DOX-high-to-negative $(\mathrm{DOXH}-)$ time course, as previously described ${ }^{10}$. For the DOXH time course, cells were maintained in DOX-high culture $\left(1.5 \mu \mathrm{g} \mathrm{ml}^{-1}\right)$. For the DOXL - time course, cells were passaged on day 8 and half of the cells were transitioned to a DOX-low level (either $5 \mathrm{ng} \mathrm{ml}^{-1}$ ). DOX-low-treated cells subsequently underwent passaging and DOX removal on day 14. For the DOXH - time course, DOX-high cells were transitioned to DOX - culture.

For derivation of EpiSCs from iPSCs and ESC controls, cells were maintained either in standard ESC culture (serum + LIF), 2i culture supplemented with $3 \mu \mathrm{M}$ CHIR99021 and $1 \mu \mathrm{M}$ PD0325901 (Reagents Direct), or standard EpiSC culture, as previously described ${ }^{25}$. Following 18 days of culture in these conditions, with regular passaging, the cells were sampled for FACS and qPCR analyses.

Cells were incubated in a humidified $5 \%(\mathrm{v} / \mathrm{v}) \mathrm{CO}_{2}$ air environment at $37^{\circ} \mathrm{C}$.

Flow cytometry and immunocytochemistry staining. Antibodies used for flow cytometry are listed in Supplementary Table 1. Surface stainings for flow cytometry were performed in the presence of 7AAD (Molecular Probes) or LIVE/DEAD Fixable Near-IR Dead Cell Stain (Life Technologies), and populations were gated on live cells. Flow cytometry was conducted on an LSRFortessa (BD Biosciences) For cell sorting, cells labelled with antibodies above and sorted using a FACSAria flow cytometer (BD Biosciences) or MoFlo Astrios (Beckman Coulter). Analysis was performed on FACSDiva (BD Biosciences) as well as FlowJo (Tree Star).

Immunocytochemistry stainings were performed by fixing cells in PBS containing $4 \%(\mathrm{v} / \mathrm{v})$ formaldehyde. Cells were permeabilized in PBS containing $0.1 \%(\mathrm{v} / \mathrm{v})$ Triton X-100 and subsequently blocked in PBS containing $10 \%(\mathrm{v} / \mathrm{v})$ donkey serum. Samples were incubated with primary and secondary antibodies in PBS containing $1 \%(\mathrm{w} / \mathrm{v})$ BSA and imaged using a confocal microscope (FV1000 laser scanning confocal; Olympus). Images represent the $\mathrm{z}$-stack projection of confocal optical sections. EdU cell proliferation assays were performed according to the manufacturer's protocol using the Click-iT EdU Pacific Blue Flow Cytometry Assay Kit (Invitrogen).

Quantitative PCR analysis. Total RNA was extracted from cells using Qiagen RNAeasy miniprep columns according to the manufacturer's protocol. Total RNA was used to generate cDNA using Superscript III reverse transcriptase (Invitrogen) according to the manufacturer's instructions. Generated cDNA was mixed with respective primers and SYBR green mix (Roche, Sigma) and run on an Applied Biosystems 7900 HAT real-time PCR machine. Relative expression of described genes was determined by the delta-delta cycle threshold $\left(C_{\mathrm{t}}\right)$ method with the expression of Gapdh (or GAPDH) as an internal reference. Primer sequences are listed in Supplementary Table 2.

Differentiation protocols. Differentiation was conducted as previously reported ${ }^{19}$ Mesoderm and endoderm differentiation was carried out by dissociating iPSC and seeding in low-adhesion plates at a density of $1 \times 10^{6}$ cells per $10 \mathrm{ml}$ in DMEM containing $15 \%(\mathrm{v} / \mathrm{v})$ FBS, $1 \%(\mathrm{v} / \mathrm{v})$ penicillin and streptomycin, $2 \mathrm{mM}$ Glutamax, $0.1 \mathrm{mM}$ BME and $0.1 \mathrm{mM}$ NEAA. Cells were cultured for 4 days on an orbital 
shaker (65 r.p.m.) with medium exchange on day 2. After 4 days, suspension aggregates were seeded on gelatin-coated tissue-culture plates and cultured for another 5 days before staining with antibodies.

Ectoderm differentiation was performed by trypsinizing and plating iPSCs at $5 \times 10^{5}$ cells per $10 \mathrm{ml}$ in SFEB medium (Glasgow minimum essential medium supplemented with $5 \%(\mathrm{v} / \mathrm{v})$ knockout serum replacement, $0.1 \mathrm{mM} \mathrm{NEAA}, 1 \mathrm{mM}$ sodium pyruvate, $1 \%(\mathrm{v} / \mathrm{v})$ penicillin and streptomycin and $0.1 \mathrm{mM} \mathrm{BME})$. Cells were cultured for 3 days in low-adherence plates. On day 3, cells were re-fed by replacing $70 \%$ of the medium and were cultured for another 2 days. Spheres were transferred intact to Geltrex-coated six-well plates and incubated for 5 days in N2B27 medium (DMEM with F12 and Neurobasal medium supplemented with B27, N2 supplements, $0.005 \%(\mathrm{w} / \mathrm{v}) \mathrm{BSA}$ and $1 \mathrm{mM}$ sodium pyruvate) before being stained with antibodies.

Human ESC cultures and induction to naive state. H9 hESCs were obtained from the WiCell Research Institute. HES2 hESCs were provided by G. Keller (McEwen Centre for Regenerative Medicine/University Health Network). HES2 and H9 cells were cultured on Geltrex LDEV-Free Reduced Growth Factor Basement Membrane Matrix (Life Technologies)-coated plates in Nutristem hESC XF Culture Media (Biological Industries), supplemented with $1 \times$ PenicillinStreptomycin (Life Technologies). H9 cells were passaged 1:12-1:24 every 5-6 days and were disassociated into small clumps using $0.1 \%$ collagenase IV (Invitrogen). HES2 cells were passaged 1:12-1:24 every 5-6 days and were dissociated to single cells using TrypLE Express (Life Technologies). All cell-line stocks were confirmed negative for mycoplasma contamination.

To convert H9 and HES2 hESCs to a naive state, primed hESCs were passaged on feeder layers of irradiated mouse embryonic fibroblasts in NHSM media as described previously ${ }^{11}$ with modifications recommended by J. Hanna. In brief, NHSM media contained Knockout DMEM (Life Technologies), 15\% Knockout Serum Replacement (Life Technologies), $1 \times$ glutamax (Life Technologies), $1 \times$ NEAAs (Life Technologies), $1 \times$ penicillin-streptomycin (Life Technologies). Added to this base media were $12.5 \mu \mathrm{g} \mathrm{ml}^{-1}$ Insulin (Sigma), $20 \mathrm{ng} \mathrm{ml}^{-1}$ human Leukemia Inhibitory Factor (made in-house $\mathrm{e}^{11}$ ), $20 \mathrm{ng} \mathrm{ml}^{-1}$ human LR3-IGF1 (prospec), $2 \mathrm{ng} \mathrm{ml}^{-1}$ tgfb1 (RnD), $12 \mathrm{ng} \mathrm{ml}^{-1}$ fgf2 (peprotech), $1 \mu \mathrm{M}$ PD0325901 (Reagents Direct), $3 \mu \mathrm{M}$ CHIR99021 (Reagents Direct), $5 \mu \mathrm{M}$ SP600125 (Santa Cruz Biotechnology), $2 \mu \mathrm{M}$ BIRB796 (Cayman Chemical), $5 \mu \mathrm{M}$ Y-27632 (Reagents Direct) and 0.4 $\mu$ M LDN193189 (Cayman Chemical). Subsequent experiments involving naive induction were conducted with an altered induction cocktail in which LDN and IGF1 were removed and the following reagents were added: $0.25 \mathrm{mg} \mathrm{ml}^{-1}$ Albumax (Invitrogen), $1 \times \mathrm{N} 2$ supplement (Life Technologies), $500 \mu \mathrm{g} \mathrm{ml}^{-1}$ Ascorbic acid (Sigma) and $1 \mu \mathrm{M}$ Go6983 (Tocris Biosciences). HES2 cells were passaged 1:30 every 4-5 days, and H9 cells were passaged 1:30 every 5-6 days on irradiated MEF-coated plates and were dissociated into single cells using TrypLE Express (Life Technologies).

Statistical and data analysis. Results were expressed as mean \pm s.d. from replicates indicated in figure legends. Statistical tests were conducted as reported for each figure. A minimum sample replicate size of $n=3$ was used for all data analyses to allow for appropriate statistical testing. Calculations for statistical significance were performed using Excel and MATLAB software. For most samples, a Student's $t$-test was used for pairwise comparison of an experimental condition of interest compared to control. Hierarchical clustering of gene expression data was conducted using the clustergram command in MATLAB. PCA and K-means clustering analysis of surface proteome data in reprogramming time course was performed using R.

\section{References}

1. Takahashi, K. \& Yamanaka, S. Induction of pluripotent stem cells from mouse embryonic and adult fibroblast cultures by defined factors. Cell 126, 663-676 (2006).

2. Park, I.-H. et al. Reprogramming of human somatic cells to pluripotency with defined factors. Nature 451, 141-146 (2007).

3. Samavarchi-Tehrani, P. et al. Functional genomics reveals a BMP-driven mesenchymal-to-epithelial transition in the initiation of somatic cell reprogramming. Cell Stem Cell 7, 64-77 (2010).

4. Polo, J. M. et al. A molecular roadmap of reprogramming somatic cells into iPS cells. Cell 151, 1617-1632 (2012).

5. Hansson, J. et al. Highly coordinated proteome dynamics during reprogramming of somatic cells to pluripotency. Cell Rep. 2, 1579-1592 (2012).

6. O'Malley, J. et al. High-resolution analysis with novel cell-surface markers identifies routes to iPS cells. Nature 499, 88-91 (2013).

7. Woltjen, K. et al. PiggyBac transposition reprograms fibroblasts to induced pluripotent stem cells. Nature 458, 766-770 (2009).

8. Golipour, A. et al. A late transition in somatic cell reprogramming requires regulators distinct from the pluripotency network. Stem Cell 11, 769-782 (2012).

9. Tonge, P. D. et al. Divergent reprogramming routes lead to alternative stem cell states. Nature 516, 192-197 (2012).
10. Hussein, S. M. I. et al. Genome-wide characterization of the routes to pluripotency. Nature 516, 198-206 (2012).

11. Gafni, O. et al. Derivation of novel human ground state naive pluripotent stem cells. Nature 504, 282-286 (2013).

12. Wollscheid, B. et al. Mass-spectrometric identification and relative quantification of N-linked cell surface glycoproteins. Nat. Biotechnol. 27, 378-386 (2009).

13. Thompson, A. et al. Tandem mass tags: a novel quantification strategy for comparative analysis of complex protein mixtures by MS/MS. Anal. Chem. 75, 1895-1904 (2003).

14. Benevento, M. et al. Proteome adaptation in cell reprogramming proceeds via distinct transcriptional networks. Nature 5, 5613 (2014).

15. Smith, L. M. \& Kelleher, N. L. Proteoform: a single term describing protein complexity. Nat. Methods 10, 186-187 (2013).

16. Thomas, P. D. PANTHER: a library of protein families and subfamilies indexed by function. Genome Res. 13, 2129-2141 (2003).

17. Mi, H. The PANTHER database of protein families, subfamilies, functions and pathways. Nucleic Acids Res. 33, D284-D288 (2004).

18. Kuhn, R. M. et al. The UCSC Genome Browser Database: update 2009. Nucleic Acids Res. 37, D755-D761 (2009).

19. Fluri, D. A. et al. Derivation, expansion and differentiation of induced pluripotent stem cells in continuous suspension cultures. Nat. Methods 9, 509-516 (2012).

20. Hou, P. et al. Pluripotent stem cells induced from mouse somatic cells by smallmolecule compounds. Science 341, 651-654 (2013).

21. Carey, B. W., Markoulaki, S., Beard, C., Hanna, J. \& Jaenisch, R. Single-gene transgenic mouse strains for reprogramming adult somatic cells. Nat. Methods 7, 56-59 (2010).

22. Tonge, P. D. et al. Divergent reprogramming routes lead to alternative stem-cell states. Nature 516, 192-197 (2014)

23. Nishimura, K. et al. Manipulation of KLF4 expression generates iPSCs paused at successive stages of reprogramming. Stem Cell Rep. 3, 915-929 (2014).

24. Rugg-Gunn, P. J. et al. Cell-surface proteomics identifies lineage-specific markers of embryo-derived stem cells. Dev. Cell 22, 887-901 (2012).

25. Onishi, K., Tonge, P. D., Nagy, A. \& Zandstra, P. W. Microenvironmentmediated reversion of epiblast stem cells by reactivation of repressed JAKSTAT signaling. Integr. Biol. (Camb.) 4, 1367-1376 (2012).

26. Toyooka, Y., Shimosato, D., Murakami, K., Takahashi, K. \& Niwa, H. Identification and characterization of subpopulations in undifferentiated ES cell culture. Development 135, 909-918 (2008).

27. Hayashi, K., Lopes, S. M., Tang, F. \& Surani, M. A. Dynamic equilibrium and heterogeneity of mouse pluripotent stem cells with distinct functional and epigenetic states. Cell Stem Cell 3, 391-401 (2008).

28. Takahashi, K. et al. Induction of pluripotent stem cells from adult human fibroblasts by defined factors. Cell 131, 861-872 (2007).

29. $\mathrm{Yu}$, J. et al. Induced pluripotent stem cell lines derived from human somatic cells. Science 318, 1917-1920 (2007).

30. Nichols, J. \& Smith, A. Naive and primed pluripotent states. Cell Stem Cell 4, 487-492 (2009).

31. Yan, L. et al. Single-cell RNA-Seq profiling of human preimplantation embryos and embryonic stem cells. Nat. Struct. Mol. Biol. 20, 1131-1139 (2013).

32. Theunissen, T. W. et al. Systematic identification of culture conditions for induction and maintenanceof naive human pluripotency. Stem Cell 15, 471-487 (2014).

33. Takashima, Y. et al. Resetting transcription factor control circuitry toward ground-state pluripotency in human. Cell 158, 1254-1269 (2014).

34. Sammar, M., Gulbins, E., Hilbert, K., Lang, F. \& Altevogt, P. Mouse CD24 as a signaling molecule for integrin-mediated cell binding: functional and physical association with src-kinases. Biochem. Biophys. Res. Commun. 234, 330-334 (1997).

35. Goode, I., Xu, H. \& Ildstad, S. T. Regulatory B cells: the new 'it' cell. Transplant. Proc. 46, 3-8 (2014).

36. Quan, M., Wang, P., Cui, J., Gao, Y. \& Xie, K. The roles of FOXM1 in pancreatic stem cells and carcinogenesis. Mol. Cancer 12, 159 (2013).

37. Magnaldo, T. \& Barrandon, Y. CD24 (heat stable antigen, nectadrin), a novel keratinocyte differentiation marker, is preferentially expressed in areas of the hair follicle containing the colony-forming cells. J. Cell Sci. 109, 3035-3045 (1996).

38. Fang, X., Zheng, P., Tang, J. \& Liu, Y. CD24: from A to Z. Cell Mol. Immunol. 7, 100-103 (2010).

39. Shirasawa, T. et al. Gene expression of CD24 core peptide molecule in developing brain and developing non-neural tissues. Dev. Dyn. 198, 1-13 (1993).

40. Lee, T. K. W. et al. CD24(+) liver tumor-initiating cells drive self-renewal and tumor initiation through STAT3-mediated NANOG regulation. Cell Stem Cell 9, 50-63 (2011).

41. Smith, S. C. et al. The metastasis-associated gene CD24 is regulated by Ral GTPase and is a mediator of cell proliferation and survival in human cancer. Cancer Res. 66, 1917-1922 (2006). 
42. Baumann, P. et al. CD24 interacts with and promotes the activity of c-src within lipid rafts in breast cancer cells, thereby increasing integrin-dependent adhesion. Cell Mol. Life Sci. 69, 435-448 (2012).

43. Lee, K.-M. et al. CD24 regulates cell proliferation and transforming growth factor $\beta$-induced epithelial to mesenchymal transition through modulation of integrin $\beta 1$ stability. Cell Signal. 24, 2132-2142 (2012).

44. Wang, L. et al. Intracellular CD24 disrupts the ARF-NPM interaction and enables mutational and viral oncogene-mediated p53 inactivation. Nat. Commun. 6, 5909 (2015).

45. Tesar, P. J. et al. New cell lines from mouse epiblast share defining features with human embryonic stem cells. Nature 448, 196-199 (2007).

46. Le, T. L., Yap, A. S. \& Stow, J. L. Recycling of E-cadherin: a potential mechanism for regulating cadherin dynamics. J. Cell Biol. 146, 219-232 (1999).

47. Marelli-Berg, F. M., Peek, E., Lidington, E. A., Stauss, H. J. \& Lechler, R. I. Isolation of endothelial cells from murine tissue. J. Immunol. Methods 244, 205-215 (2000).

48. Ware, C. B. et al. Derivation of naive human embryonic stem cells. Proc. Natl Acad. Sci. USA 111, 4484-4489 (2014).

49. Kislinger, T. PRISM, a Generic Large Scale Proteomic Investigation Strategy for Mammals. Mol. Cell. Proteomics 2, 96-106 (2003).

\section{Acknowledgements}

We thank Kento Onishi for providing EpiSC samples. We also thank Dr Jeff Wrana and Dr Dan Trcka for providing secondary DOX-inducible reprogramming system to allow further validation of our findings. We would also like to acknowledge the assistance and support of laboratory colleagues and collaborators. This work has been supported by a GL2 grant from Ontario Government (AN) and a CIHR Grant to P.W.Z. N.S. is the recipient of the NSERC Vanier Canada Graduate Scholarship. A.N. is Tier 1 Canada Research Chair in Stem Cells and Regeneration. P.W.Z. is the Canada Research Chair in Stem Cell Bioengineering.

\section{Author contributions}

N.S. and P.W.Z. conceived and designed the experiments, and wrote the manuscript. A.N. contributed to study design. N.S. performed all validation flow cytometry, live imaging and cell culture work. C.A.W. performed mass spectrometry analysis, with input from B.W. Global proteomics data were collected and analysed by J.M., M.B. and A.J.R.H. N.S. and P.D.T. performed the qPCR analysis. A.Y.-K. performed data mining of surface proteome analysis. Y.L. and J.M. performed all hESC culture and naive induction with input from J.H.H. S.M.I.H. contributed to data analysis. M.C.P. and J.E. created secondary reprogramming lines from embryos derived by tetraploid complementation. M.L. contributed to sample preparation for validation sequencing analysis, with support from I.M.R.

\section{Additional information}

Accession codes: The mass spectrometry proteomics data have been deposited to the ProteomeXchange Consortium (http://proteomecentral.proteomeexchange.org) via the PRIDE partner repository with the data set identifier PXD001456.

Supplementary Information accompanies this paper at http://www.nature.com/ naturecommunications

Competing financial interests: The authors declare no competing financial interest.

Reprints and permission information is available online at http://npg.nature.com/ reprintsandpermissions/

How to cite this article: Shakiba, N. et al. CD24 tracks divergent pluripotent states in mouse and human cells. Nat. Commun. 6:7329 doi: 10.1038/ncomms8329 (2015).

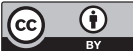

This work is licensed under a Creative Commons Attribution 4.0 International License. The images or other third party material in this article are included in the article's Creative Commons license, unless indicated otherwise in the credit line; if the material is not included under the Creative Commons license, users will need to obtain permission from the license holder to reproduce the material. To view a copy of this license, visit http://creativecommons.org/licenses/by/4.0/ 